\title{
Effect of stimulus location and response measure on an instrumental avoidance conditioning paradigm in spinal rat
}

\author{
F. SPADA and A. A. BUERGER \\ Departments of Physical Medicine and Rehabilitation and Physiology \\ California College of Medicine, University of California, Irvine, California 92717
}

\begin{abstract}
We have previously demonstrated the acquisition and retention of instrumental avoidance conditioning of leg withdrawal in spinal rat using a yoked control and footshock in the paradigm of Horridge (1962); when shock to the foot is a function of leg position, acquisition and retention occur if either foot insertion rate or percentage of time with the leg elevated is used as a response measure. The present experiment indicates that, when shock is delivered to the base of the tail, the experimental effect is weaker and less consistent. Insertion rate yields no evidence of acquisition or retention. If percentage of time with the leg elevated is used as a response measure, the results might be consistent with acquisition and retention during the early portions of the testing period. However, sensitization is a more parsimonious explanation of these results.
\end{abstract}

The ability of spinal animals to learn has been the subject of research for some decades (Dykman \& Shurrager, 1956; Kellogg, Deese, Pronko, \& Feinberg, 1947; Patterson, Cegavske, \& Thompson, 1973). Evidence is accumulating that chronic spinal rats (Buerger \& Fennessy, 1971) and frogs (Farel $\&$ Buerger, 1972) can acquire and retain an instrumental avoidance response when the paradigm and controls of Horridge (1962) are used. The data presented below further elucidate the capacity for instrumental avoidance conditioning of low spinal rats. The study evolved from the findings of Buerger and Fennessy $(1970,1971)$, which recently have been independently confirmed (Chopin, 1974). These studies provide convincing evidence that the spinal rat demonstrates acquisition and retention of an instrumental shock-avoidance task in which the response is hind-limb flexion and in which shock to the hind foot is contingent upon its extension. These experiments have recently been reviewed (Buerger \& Chopin, 1976).

We were interested in the extent to which different stimuli and responses could be associated within the spinal cord by instrumental conditioning. This type of experiment might yield information about the number and location of spinal neurons which are susceptible to this type of procedure. Also, if two or more potent inputs could be located, a differential conditioning control would be possible. In the present experiment, using the Horridge paradigm, we attempted to determine if inputs along neural pathways, distinct from those previously used, could

This research was partially supported by the General Research Support Funds of the California College of Medicine and by SRS Grant No. 44-P-45065/9-11. function as discriminative stimuli for operant conditioning.

\section{METHOD}

The essentials of both the surgical techniques and the methods of recording responses have been previously reported (Buerger \& Fennessy, 1971; Farel \& Buerger, 1972). Only an abbreviated description, emphasizing the small differences, is presented here. In brief, the spinal cords of six pairs of $250-350-g$ Sprague-Dawley rats were transected between the 11 th and 12th thoracic vertebrae, under pentobarbital anesthesia $(40 \mathrm{mg} / \mathrm{kg})$, supplemented with ether when necessary. After a laminectomy of these vertebrae, the spinal cord, as well as all dorsal and ventral roots, were carefully and completely cut. Bleeding was controlled with cotton or gelatin sponge soaked in saline or thrombin solution. After the muscle and skin were closed in layers, 250,000 units of penicillin-G (Bicillin, Wyeth Laboratories) were injected intramuscularly. Sickly animals were never used in the experiment. Both animals in the same pair were allowed identical recovery periods. Because an animal in one of the pairs became sick during its first run, that pair of animals was trained and tested only once. Hence (as described below), five of the six pairs were run through the experiment twice; these six pairs of rats yielded 11 runs through the experiment.

After a 3- to 14-day period, one member of each pair of rats was randomly designated as an experimental or control animal. They were firmly secured in wire mesh tubes supported in chemist's clamps and frames (Flexframe, Fisher Scientific, Indiana, Pennsylvania). The tubes allowed the hindquarters of the rats to hang freely. The hindquarters of each rat were firmly fastened in a burette clamp, modified such that opposing pins could be clamped on the pelvis. Thus the pelvis and caudal thorax were fixed while the hind limbs were free to move. After one leg was taped out of the way, an insulated stainless steel touch electrode (approximately $.25 \mathrm{~mm}$ in diam) was inserted through a fold of skin over the metacarpal bones of the unrestrained leg until it hung 5 or more centimeters below the foot. Shock electrodes of stainless steel wound clips or needles were inserted on the base of the tail. These electrodes were connected to a Grass S88 stimulator by the relay and circuit described 


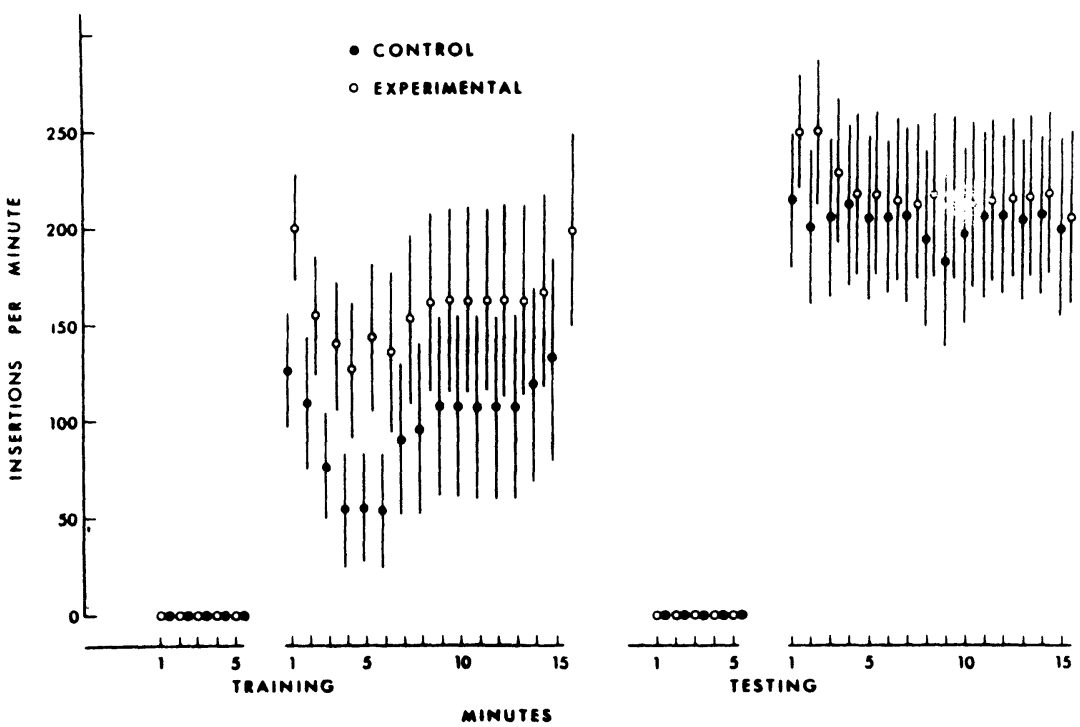

Figure 1. Insertion rate. Insertions (into the fluid) per minute are plotted vs. time for the training and testing periods of an instrumental avoidance task with a yoked control. The points are means and standard errors for the pooled results of six pairs of animals, five run through the paradigm twice in a counterbalanced design, and the sixth run through once. Note that this measure yields no statistical evidence for acquisition or retention, because the control animals always have lower insertion rates than the experimental animals.

below. When the relay closed, stimuli were delivered at 6 pulses/ $\mathrm{sec}$; each was $1 \mathrm{msec}$ in duration and $5-10 \mathrm{~mA}$ in strength. The intensity of the shock was increased until it was sufficient to elicit removal of the foot electrode from the solution due to leg flexion.

Each rat was suspended over a basin of water, and the rat or basin was raised or lowered until the touch electrode was 1-3 mm below the water's surface; however, the electrodes of each pair were always immersed on equal distance. A second electrode was submerged in the water basin. When both the touch and basin electrodes were in the water, an insensible current passed through this completed circuit. This current was amplified such that it closed a relay, allowing stimuli to pass from the Grass S88 stimulator to the shock electrodes. As was reported by Buerger and Fennessy (1971), extension of the shocked foot was prevented by tying a taut ligature between the metacarpals of the shocked foot and some point anterior to the rat.

Whenever the electrode was immersed in the water of the basin, the electronic circuitry also produced a voltage change which was recorded through the driver amplifier of a Grass Model 7 polygraph. This record was used for later analysis.

A modified form of the Horridge paradigm was used; it is very similar to that employed by Farel \& Buerger (1972). After a baseline period (designed to assure that the unstimulated levels of each pair of animals were essentially identical), a 15-min training period commenced; during this period, both the experimental and control animals were shocked whenever the experimental animal lowered its touch electrode below the level of the fluid. The fluid bath and the touch electrode of the experimental animal therefore formed an electrical switch for delivery of shocks to both the experimental and control animals. The 15-min training period was followed by a second $5-10$-min baseline period in which the depth to which each foot electrode was'submersed was again set equal. The second baseline or resting period was in turn succeeded by a 15 -min testing period in which the position of each animal's touch electrode determined if that animal was shocked. In short, during the testing period, each animal's touch electrode may be considered an electrical switch delivering pulses to the shock electrodes of that animal, whereas during the training period, the touch electrode of the experimental animal may be considered an electrical switch delivering pulses to the shock electrodes of both animals.

With the exception noted above, the rats of each pair were put through this series of periods twice, one as an experimental and one as a control animal; the right hind limb was used for one run and the left for the other.
As has been described elsewhere (Buerger \& Chopin, 1976), this reversal of function within the paradigm tends to offset the consistent bias of the yoked control design discussed by Church (1964; see also Teyler, Baum, \& Patterson, 1975). Because there were no detectable differences between the first run with the right legs and the second run with the left legs, they were pooled in presenting the results: The Mann-Whitney $U$ statistic was used in this test and, except as noted, in all other comparisons reported here.

\section{RESULTS}

The effects of the modified Horridge paradigm have been plotted in Figures 1 and 2, using two different dependent variables: rate of electrode insertion, as was used in Buerger and 'Fennesy (1971) and percentage of time in the fluid, as was used by Farel and Buerger (1972).

\section{Baseline and Resting Periods}

By either measure, no significant differences between the control and experimental animals was apparent during these periods.

\section{Training Period}

If rate of electrode insertion was used as the dependent variable, the insertion rates of the experimentals was higher than that of the controls (Figure 1, Training). This difference was greater during the early minutes of the period. For example, except for the second minute, the standard errors of the means did not overlap for the first $6 \mathrm{~min}$, but did so later in the period. Because one would expect the experimental animals to lower their insertion rates if they had learned to avoid shock by sustained leg flexion, this data would not be considered consistent with the interpretation that the experimental animals had acquired this instrumental response. 


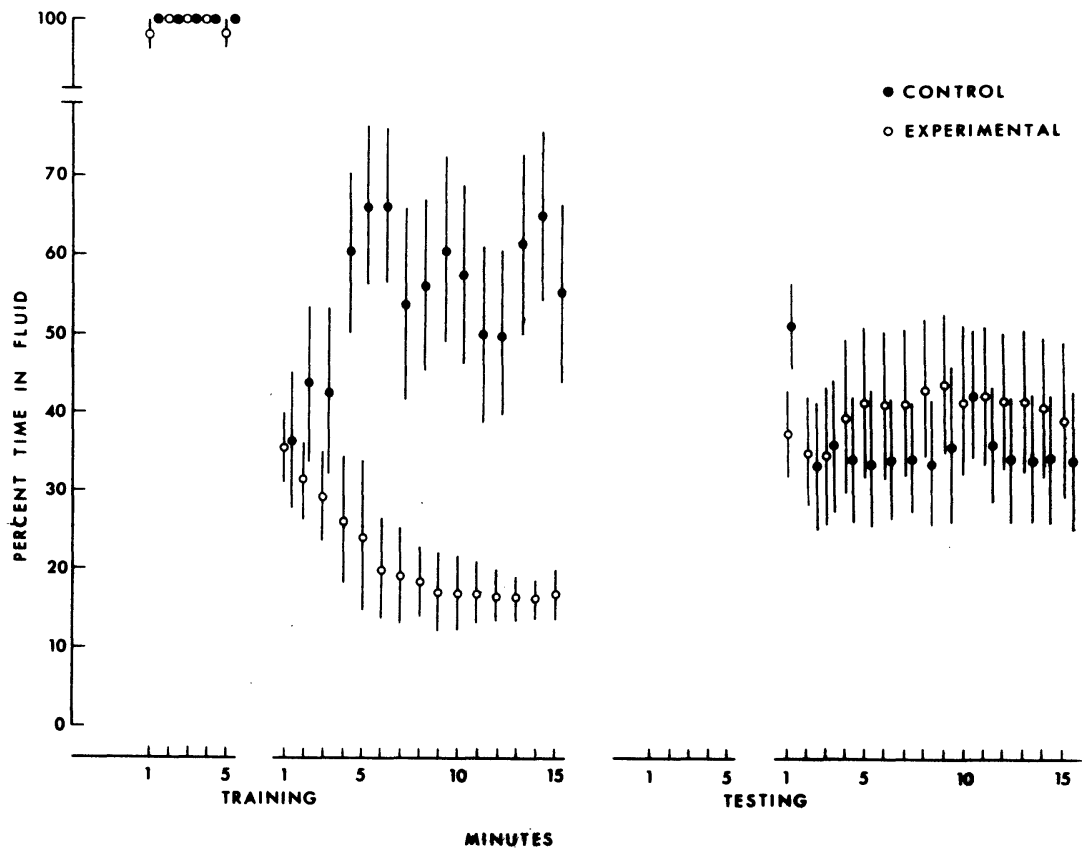

Figure 2. Percentage of time in fluid. Percent of time below the fluid level is plotted vs. time as in Figure 1. Note that during all of training and early testing, this measure yields statistical evidence of acquisition and retention.

If percentage of time in the fluid is used, a different conclusion is possible (Figure 2, Training). The controls spent more time with their foot electrodes lowered into the fluid than did the experimental animals $(p<.01)$. In addition, with the passage of time during this period, note that this measure tended to increase for the control animals and decrease for the experimental animals. Both of these observations might be interpreted as evidence for acquisition of instrumentally conditioned response by spinal animals. However, these results could also be due to sensitization, providing the sensitizing effect was more potent when tailshock was a function of foot position than when it was not related to foot position. The slight tendencies for the foot insertion rate of the experimentals to be greater than that of the controls, and to increase during the training period, might also be due to a form of position-contingent sensitization.

\section{Testing Period}

There is also a difference between the two response measures during the testing period. If insertion rate is used as a measure, no evidence of retention emerges. Furthermore, because the insertion for experimental rats is actually above that for the controls (although the standard errors markedly overlap), the results using insertion rate suggest no retention.

If percentage of time in the fluid is the response measure, the performance of the experimental animals is significantly less $(p<.05)$ than that of the controls for the first minute of testing. This result could be interpreted as consistent with retention of this instrumental avoidance response or with a prolonged sensitization. Because the percentage of time in the fluid at the beginning of the testing period is virtually identical to that during the beginning of the training period, sensitization is the most probable explanation. However, in most of the latter minutes of the testing period, the control animals tended to spend a smaller percentage of time in the fluid than did the experimentals. These differences are not significant according to a Mann-Whitney $U$ test on the individual data $(p<.32)$, although a sign test on the means for each minute is significant at the $p<.05$ level. Thus, the results from the latter minutes suggest that neither retention nor sensitization occurred, and may be consonant with those observed when insertion rate is the response measure.

\section{DISCUSSION}

Previous evidence indicates that spinal vertebrates learn to shorten a shocked leg, thereby terminating the delivery of shock to that leg, when the shock is delivered to the foot (Buerger \& Fennessy, 1970, 1971; Farel \& Buerger, 1972). In other words, instrumental avoidance conditioning occurs when shock is delivered to the foot. The present experiment attempted to determine if another location, in addition to the foot, was an adequate site of stimulation for this type of conditioning. The results depended on the response measure used. If insertion rate is used as the response measure when shock is delivered to the base of the tail, no evidence for acquisition or retention is apparent (Figure 1). If percent of time in the fluid was used as the response 
measure, different results are observed; there is a significant difference between the experimental animals during the training period and during the first minute of the testing period. These observations might be explained as acquisition and retention of an instrumental avoidance task or as a relatively long-lasting sensitization which is more potent when shock is contingent upon foot position. Because there is no significant difference between the experimental animals at the beginning of the training and testing periods, it seems probable that positioncontingent sensitization is the more parsimonious explanation. In short, different response measures appear to yield different results.

The data also suggest that, during instrumental avoidance conditioning, the caudal segments of the transected vertebrate spinal cord are not equally efficient in associating all sensory inputs (e.g., shock to tail base or foot) with the same output (e.g., leg withdrawal). Consider the results when using the Horridge paradigm in rats. Although pairing one input (footshock) with a given output (foot withdrawal) yields strong evidence for instrumental avoidance conditioning (Buerger \& Fennesy, 1971; Chopin, 1974), pairing a different input (shock to the base of the tail) with the same output yields equivocal results which at least partially depend upon the response measure used. However, insertion rate is a relatively indirect measure which might or might not reflect behavioral co-relates of plasticity. Consider the extreme case in which the control animal leaves his electrode in the fluid throughout a 15-min period, and the experimental animal holds his leg out except for one or two shocks. Using insertion rate as a measure, one would not conclude that learning occurred. Hence, it may not be surprising that the insertion rate data led to a conclusion less supportive of retention than the data for percent time in fluid.

These results may help to explain the discrepancies between the results of Kellogg and his associates (e.g., Kellogg et al., 1947) and Shurrager and his group (e.g., Dykman \& Shurrager, 1956). These groups both studied classical conditioning paradigms in spinal carnivores. In their early experiments, they used diverse locations for skin stimulation, and they used different response definitions in all their experiments. One reported negative results, the other positive. The results presented here suggest that some of the long-standing differences between Shurrager's and Kellog's groups might be partially due to limitations on the associative ability with the transected spinal cord; transected animals may yield significantly different results when different input paths or response measures are employed.

Why is it that animals which appear to show good acquisition show much worse performance after a rest period, especially during the latter portions of the testing period? There is an interesting parallel with the data from insect preparations of Hoyle (1965) and R. B. Stein (personal communication). During instrumental avoidance conditioning of the firing rate of single cells in insect preparations, both these investigators found that, while it is possible to drive up the cell firing rate for a period of time, performance frequently seemed to collapse after a large number of shocks had been administered. As Church (1964, 1972; Campbell \& Church, 1967; Teyler, Baum, \& Patterson, 1975) has pointed out, some of the positive results of the Horridge paradigm can be accounted for in terms of sensitization and response variables, without the necessity of invoking instrumental conditioning. (However, as Buerger and Chopin [1976] have discussed, all the positive results of a counterbalanced Horridge paradigm can be best accounted for by instrumental avoidance conditioning). The fact that the results described above can be explained as a form of long-lasting position-contingent sensitization is an indication of the importance of the arguments raised by Church. In summary, because the Horridge paradigm produces a weaker and more inconsistent effect when shock is delivered to the base of the tail than when it is delivered to the foot, it is possible to account for the present data in terms of sensitization and response variables.

There are a variety of explanations for these results. For example, note that the shock was delivered at $6 / \mathrm{sec}$ or $360 / \mathrm{min}$. If each shock elicited a flexion, the maximum insertion rate would be $360 / \mathrm{min}$ with an interval of about $166 \mathrm{msec}$. The initial rate of about $150-200 / \mathrm{min}$ for the experimentals indicates that they were receiving about half the possible shocks. The leg was, on the average, receiving a shock, raising and reentering the fluid just after the immediately following shock. The leg therefore tended to receive the shock after the next one. This hypothesis is supported by the fact that the time in fluid during the initial periods was about $35 \%$. If a slight sensitization occurred during the training period, it would cause the leg to raise a bit higher during flexion withdrawal and come back into the fluid slightly later, but still before the third shock. This hypothetical sensitization might cause some of the observed results, such as a lower percent time in fluid with smaller standard errors, but not change in insertion rate. If the slight sensitization disappeared during the rest period, the experimental group would return to its initial training percentage. Thus, one could account for this portion of the results without invoking retention of an instrumental avoidance response. Also, if, during the training and resting periods, the legs became fatigued from 
hanging, one could account for the fact that insertion rate jumped to $200-225 / \mathrm{min}$ while time in fluid stayed at the original level. This fatigue might cause the leg to raise less after each shock and to sometimes come down within $166 \mathrm{msec}$ to receive the next shock. Therefore, if on some trials the leg came down within $166 \mathrm{msec}$, was shocked, and immediately went up, whereas on other trials, it came down in more than $166 \mathrm{msec}$, and after the next possible shock, and waited the remainder of the interpulse interval before being shocked and going up again, the observed $40 \%$ time in fluid would result. If, meanwhile, the controls during training were sometimes shocked just before their legs were reinserted, they would have slightly lower insertion rates; they would tend toward more time in the fluid because of their tendency to insert before the experimental leg. Sensitization in this group might then make little or no difference in the measures used. And, if during testing, these animals suffered from hanging fatigue, they would have the same behavior as the experimentals.

Because the experimental animals have higher insertion rates than the control animals during the same period, they tend to hold their leg electrodes above the level of the fluid. Another possible explanation of these results is that, when shock is delivered to the base of the tail, spinal rats acquire an increased tendency to briefly flex their legs after each shock, but do not learn to maintain flexion. This increased tendency to brief leg flexion might be viewed as a form of sensitization. They might also suggest distinct neural mechanisms for the short-term phasic flexion vs. long-term tonic flexion.

Other mechanisms which might underlie these results could be suggested, but this is probably not a profitable exercise until more data can be accumulated in order to distinguish among the various possibilities. However, the evidence available does allow one to conclude that, when an instrumental avoidance paradigm is used, the spinal cord is not equally efficient in pairing all skin locations with the necessary motor output. Investigation of the neuroanatomy and/or electrophysiology of the differences between the neuronal pathways from different skin locations might yield evidence about the different mechanisms underlying these forms of behavioral plasticity.

\section{REFERENCES}

Buerger, A. A., \& Fennessy, A. Learning of leg position in chronic spinal rats. Nature, 1970, 225, 751-752.

Buerger, A. A., \& Fennessy, A. Long-term alteration of leg position due to shock avoidance by spinal rats. Experimental Neurology, 1971, 30, 195-211.

Buerger, A. A., \& Chopin, S. F. Instrument avoidance learning in spinal vertebrates. In A. Reisen \& R. F. Thompson (Eds.), Advances in psychobiology. New York: Wiley, 1976, in press.

CAMpbell, B. A., \& Church, R. M. (Eds.). Punishment and aversive behavior. New York: Appleton-Century-Crofts, 1967.

ChоріN, S. F. An experimental model system for learning and memory studies: The rat spinal cord isolated from cortical structures. PhD dissertation, Medical Center, Louisiana State University, 1974.

ChURCH, R. M. Systematic effects of random error in yoked control design. Psychological Bulletin, 1964, 62, 122-131.

Church, R. M., \& GetTy, D. J. Some consequences of the reaction to an aversive event. Psychological Bulletin, 1972, 78, 21-27.

Dykman, R. A., \& Shurrager, P. S. Successive and maintained conditioning in spinal carnivores. Journal of Comparative and Physiological Psychology, 1956, 47, 345-351.

HoYLE, G. Neurophysiological studies of "learning" in headless insects. In J. E. Treherne \& J. W. L. Beament (Eds.), The physiology of the insect central nervous system. London: Academic Press, 1965. Pp. 203-232.

HorRIDGE, A. A. Learning of leg position by headless insects. Nature, 1962, 163, 697-698.

Kellogg, W. N., Deese, J., Pronko, N. H., \& Felnberg, M. An attempt to condition the chronic spinal dog. Journal of Experimental Psychology, 1947, 37, 99-117.

Patterson, M. M., Cegavske, C. F., \& Thompson, R. F. Effects of a classical conditioning paradigm on hind limb flexor nerve response in immobilized spinal cats. Journal of Comparative and Physiological Psychology, 1973, 84, 88-97.

Teyler, T. J., Baum, W. M., \& Patrerson, M. M. Behavioral and biological issues in the learning paradigm. Physiological Psychology, 1975, 3, 65-72.

(Received for publication July 1, 1975; revision accepted March 2, 1976.) 